\title{
Segmentation of Medical Images Using Topological Concepts Based Region Growing Method
}

\author{
B. Shanthi Gowri ${ }^{1}$, Gnanambal Ilango ${ }^{2}$ \\ ${ }^{1}$ Department of Mathematics, Sri Krishna College of Engineering and Technology, Coimbatore - 641008 , \\ Tamil Nadu, India. \\ ${ }^{2}$ Department of Mathematics, Government Arts College, Coimbatore - 641 018, Tamil Nadu, India.
}

\begin{abstract}
Image segmentation is an important task in the field of image processing. Medical image segmentation plays a vital role in assisting the radiologists to visualize and analyze the region of interest in medical images. Region growing is a very useful technique for image segmentation. Region growing method of segmentation which is based on the classification of pixels into connected components by selecting a seed and grouping its neighbours with the seed based on the gray levels of the neighbours. In this article, we propose a new seeded region growing segmentation algorithm based on metric topological $\varepsilon$-neighbourhoods of different metrics and grouping criterion for segmentation of region of interest from the medical images. The qualities of segmented images are measured by the evaluation measure 'Accuracy'.
\end{abstract}

Keywords: Metrics, Region Growing, Segmentation, Topological Neighbourhoods.

\section{Introduction}

Segmentation of medical images like MRI, ultrasound image, CT, X-ray is a hotspot and focus of image processing techniques. Medical image segmentation is the most important technique to visualize the affected region of interest for the radiologists to access patients for diagnosis and treatment of diseases. Image segmentation is a process of partitioning a digital image into disjoint regions of connected pixels that are homogeneous with respect to some characteristics such as gray level or colour. Some of the image segmentation methods based on the above techniques are edge detection, thresholding, region growing, region splitting and merging, clustering, watershed algorithm etc.,

Jiang Guangyou et al., [1] introduced a threshold segmentation algorithm based on neighbourhood characteristics described in a mathematical way and obtained a good segmentation effect through the contrast. Chantal Revol et al., [2] proposed a new minimum variance region growing algorithm with a homogeneity criterion based on an adequate tuning between spatial neighbourhood and histogram neighbourhood through special morphological operations and gave the result as this algorithm is an efficient solution for difficult segmentation problems. Yi-Ta $\mathrm{Wu}$ et al., [3] developed a top-down region dividing based approach which combines the advantages of both histogram-based and region-based approaches and showed that it is efficient for segmentation without distorting the spatial structure. Maria Kallergi et al., [4] developed local thresholding and region growing algorithms and applied to digitized mammograms and proved that computerized parenchymal classification of digitized mammograms is possible and independent of exposure. Pastore, J. et al., [5] described an automatic segmentation of computerized axial tomography images with tumors by means of alternating sequential filters of mathematical morphology and connected components extraction based on continuous topology concepts. Gnanambal Ilango and R. Marudhachalam [6] presented an automatic method relevant to medical image segmentation by means of fuzzy hybrid filter for denoising and topological concepts to extract connected components and edge detection. Tamas sandor et al.,[7] described an automated procedure based on the principles of region growing and nearest neighbour classification to extract continuous areas from brain CT scans and showed that it is fast and reasonably accurate method for segmenting brain tissue. A comparative performance study of thresholding techniques for segmentation was investigated by Sang Uk Lee and Seok Yoon Chung [8] and proved that the performances are image dependent. Junchai Gao et al., [9] proposed a threshold and edge detection fused segmentation algorithm and showed that it is an effective and practical image segmentation algorithm which considers not only the adjacent uniformity but also the local contrast. M.M. Abdelsamea [10] proposed an automatic seeded region growing algorithm for cellular image segmentation and gave the result as it can overcome the oversegmentation problem and is less noisy. An automatic method of morphological segmentation and region growing to detect the centre and boundaries of the abnormal object of the medical images accurately was proposed by K. Martin Sagayam et al.,[11]. Dinesh D. Patil and Sonal G. Deore [12] compared the different image segmentation techniques like edge detection, thresholding, region growing, region splitting and merging, clustering, watershed and fast region merging methods and gave the conclusion as neither a single method is good for all types of images nor all methods are equally good for a particular type of image. 
In this article, a new seeded region growing segmentation algorithm is proposed to segment the region of interest from the medical images. This algorithm is based on metric topological $\varepsilon$ - neighbourhoods of different metrics and grouping criterion. This work is organized as follows: Section II explains the seeded region growing method of segmentation, Section III gives the basic definitions, Section IV provides the proposed segmentation algorithm, Section V explains the method of evaluation of segmentation, Section VI deals with the experimental work, Section VII discusses the result analysis, Section VIII gives the conclusion and Section IX gives the table and figures of the experimental results.

\section{Seeded Region Growing Method Of Segmentation}

Image segmentation algorithms are mainly based on two properties, detecting discontinuities and similarities. The first category is based on the abrupt changes in intensity and the second category is based on grouping the set of similar pixels satisfying predefined criterion. Region based segmentation is a classical method which comes under the second category. This method tries to extract the object that is connected based on some predefined criterion. This criterion can be based on the intensity information and edges in the image. One of the region based segmentation methods is the Seeded Region Growing method. This is a procedure that groups pixels in whole image into sub regions based on predefined criterion and processed in four steps. (i) Select a seed pixel in the original image. (ii) Select a set of similarity criterion such as gray level or colour and set up a stopping rule. (iii) Grow region by appending to the seed, those neighbouring pixels that have predefined properties similar to seed pixel. (iv) Stop region growing when no more pixels meet the criterion for inclusion in that region.

Here from the histogram of the image, the gray level of the region of interest is selected. Considering the gray level of the region of interest, a seed point is selected as a point having maximum number of metric topological $\varepsilon$-neighbourhood. For the similarity criterion, a grouping criterion is defined based on metric and gray level difference. The connected region of interest is grown by including the $\varepsilon$ - neighbours of the seed point satisfying the grouping criterion with the seed point. The qualities of the segmented region of interest from the medical images by the proposed algorithm is compared with the ground truth images and the results are analyzed.

Definition 3.1[13]

\section{Basic Definitions}

A metric on a set $X$ is a function $d: X x X \rightarrow R$ having the following properties:

i. $\quad d(x, y) \geq 0 \quad \forall x, y \in X ;$ equality holds iff $x=y$.

ii. $\quad d(x, y)=d(y, x) \quad \forall x, y \in X$.

iii. $\quad d(x, z) \leq d(x, y)+d(y, z) \forall x, y, z \in X$.

Given a metric $d$ on $X$, the number $d(x, y)$ is called as the distance between $x$ and $y$ in the metric $d$. For a given $\varepsilon>0$, consider the set $B_{d}(x, \varepsilon)=\{y: d(x, y)<\varepsilon\}=B(x, \varepsilon)$ of all points y whose distance from $x$ is less than $\varepsilon$. Here $B_{d}(x, \varepsilon)$ is called the $\varepsilon$ - ball centered at $x$. If $d$ is a metric on the set $X$, then the collection of all $\varepsilon$ - balls $B_{d}(x, \varepsilon)$ for $x \in X$ and $\varepsilon>0$, is a basis for a topology on $X$, called metric topology induced by $d$. A set $U$ is open in the metric topology induced by $d$ iff for each $y \in U$, there is a $\delta>0$ such that $B_{d}(y, \delta) \subset U$. If $X$ is a topological space, $X$ is said to be metrizable if there exists a metric $d$ on the set $X$ that induces the topology of $X$. A metric space is a metrizable space $X$ together with a specific metric $d$ that gives the topology of $X$.

Definition 3.2[14]

An image may be defined as a two-dimensional function $f(x, y)$, where $x$ and $y$ are spatial (plane) coordinates, and the amplitude of $f$ at any pair of co-ordinates $(x, y)$ is called the intensity or gray level of the image at that point. If $x, y$ and the intensity values of $f$ are all finite, discrete quantities, we call the image a digital image. A digital image is composed of a finite number of elements $(x, y)$ each of which has a particular location and value. These elements are called picture elements or pixels.

Definition 3.3[15]

Let $S^{2}=S x S \subset N x N$, where $S^{2}=\{(x, y) ; x=1,2,3, \ldots m ; y=1,2,3, \ldots n\}$ be the set of spatial co-ordinates of an image. For any metric space $\left(S^{2}, d\right)$, any $p \in S^{2}$ and any $\varepsilon>0$, consider the set 
$N_{d, \varepsilon}(p)=\{q ; d(p, q)<\varepsilon+1\} . N_{d, \varepsilon}(p)$ is called the (open) $\varepsilon$ - neighbourhood of $p$ in $\mathrm{S}^{2} . N_{d, \varepsilon}(p)$ is an open ball centered at $p$.

Definition 3.4[16]

Let $\quad p=\left(x_{1}, y_{1}\right), q=\left(\begin{array}{ll}x_{2} & y_{2}\end{array}\right) \in S^{2}$. Consider the functions

i. $\quad d_{4}: S x S \rightarrow R$, defined by $d_{4}(p, q)=\left|x_{1}-x_{2}\right|+\left|y_{1}-y_{2}\right|$. Then $\left(S^{2}, d_{4}\right)$ is a metric space. The metric $d_{4}$ is called the City - Block metric or Manhattan metric.

ii. $\quad d_{8}: S x S \rightarrow R$, defined by $d_{8}(p, q)=\max \left\{\left|x_{1}-x_{2}\right|,\left|y_{1}-y_{2}\right|\right\}$. Then $\left(S^{2}, d_{8}\right)$ is a metric space. The metric $d_{8}$ is called the Chessboard metric.

Definition 3.5[15]

For any point $p$, the 4 - neighbourhood of $p$ denoted by $N_{d_{4}, 1}(p)$ is defined as $N_{d_{4}, 1}(p)=\left\{q \in S^{2} ; d_{4}(p, q)<2\right\} . \quad N_{d_{4}, 1}(p)$ is also denoted by $N_{4}(p)$ and the 8 neighbourhood of $p$ denoted by $N_{d_{8}, 1}(p)$ is defined as $N_{d_{g}, 1}(p)=\left\{q \in S^{2} ; d_{8}(p, q)<2\right\}$. $N_{d_{8}, 1}(p)$ is also denoted by $N_{8}(p)$.

Definition 3.6[15]

For any point $p$, the $4-$ neighbours of $p$ are $N_{d_{4}, 1}(p)-\{p\}$ and the 8 - neighbours of $p$ are $N_{d_{8}, 1}(p)-\{p\}$.

Definition 3.7[15]

Let $\quad M \subseteq S x S \quad$ where $\quad M=\left\{\begin{array}{c}\{(x-k, y+k)\} \cup\{(x+k, y-k)\} ; \\ \text { where } k=0,1,2,3 \ldots .\end{array}\right\} . \quad$ Let $p=\left(x_{1}, y_{1}\right), q=\left(x_{2}, y_{2}\right) \in M \quad$. Consider the function $d_{L T}: M \rightarrow R$ defined by $d_{L T}(p, q)=\frac{1}{2}\left[\left|x_{1}-x_{2}\right|+\left|y_{1}-y_{2}\right|\right] .\left(M, d_{L T}\right) \quad$ is a metric space. For any point $p$, the $L T_{\varepsilon}$ neighbourhood of $p$ is defined as $N_{d_{L T}, \varepsilon}(p)=\left\{q ; d_{L T}(p, q)<\varepsilon+1\right\}$. Hence the $L T_{1}$ neighbourhood of $p$ is defined as $N_{d_{L T}, 1}(p)=\left\{q ; d_{L T}(p, q)<2\right\}$ and the $L T_{2}$ neighbourhood of $p$ is defined as $N_{d_{L T}, 2}(p)=\left\{q ; d_{L T}(p, q)<3\right\} . N_{d_{L T}, 1}(p)$ is also denoted by $L_{3}(p)$. The $L T_{1}$ neighbours of $p$ are $N_{d_{L T}, 1}(p)-\{p\}$ and the $L T_{2}$ neighbours of $p$ are $N_{d_{L T}, 2}(p)-\{p\}$.

Definition 3.8

Let $\quad M \subseteq S x S \quad$ where $\quad M=\left\{\begin{array}{c}\{(x+k, y-k-l)\} \cup\{(x-k, y+k-l)\} \text {; } \\ \text { where } k=0, \pm 1, \pm 2, \pm 3 \ldots \text { and } l=0,1,2, \ldots\end{array}\right\}$.

Let $p=\left(x_{1}, y_{1}\right), q=\left(x_{2}, y_{2}\right) \in M$. Consider the function $d_{\Delta^{T}}: M \rightarrow R$ defined by $d_{\Delta^{T}}(p, q)=\max \left\{\left|x_{1}-x_{2}\right|,\left|y_{1}-y_{2}\right|\right\} \quad . \quad\left(M, d_{\Delta^{T}}\right) \quad$ is a metric space. For any point $p$, the ${ }_{\Delta} T_{\varepsilon}$ neighbourhood of $p$ is defined as $N_{d}{ }_{{ }_{\Delta} T^{\varepsilon}}(p)=\left\{q ; d_{\Delta^{T}}(p, q)<\varepsilon+1\right\}$. Hence the ${ }_{\Delta} T_{1}$ neighbourhood of $p$ is defined as $N_{d}{ }_{\Delta T^{1}}(p)=\left\{q ; d_{\Delta^{T}}(p, q)<2\right\}$ and the ${ }_{\Delta} T_{2}$ neighbourhood of $p$ is defined as $N_{d}{ }_{\Delta T^{2}}(p)=\left\{q ; d{ }_{\Delta T}(p, q)<3\right\}$. The ${ }_{\Delta} T_{1}$ neighbours of $p$ are $N_{d}{ }_{\Delta^{T} T^{1}}(p)-\{p\}$ and the ${ }_{\Delta} T_{2}$ neighbours of $p$ are $N_{d}{ }_{\Delta T^{\prime 2}}(p)-\{p\}$.

Definition 3.9

Let $M \subseteq S x S$ where $M=\left\{\begin{array}{c}\{(x+k, y-k+l)\} \cup\{(x-k, y+k+l)\} ; \\ \text { where } k=0, \pm 1, \pm 2, \pm 3 \ldots \text { and } l=0,1,2, \ldots\end{array}\right\}$ Let
$p=\left(x_{1}, y_{1}\right), q=\left(x_{2}, y_{2}\right) \in M \quad$. Consider the function $d_{\Delta_{T}}: M \rightarrow R$ defined by 
$d_{\Delta_{T}}(p, q)=\max \left\{\left|x_{1}-x_{2}\right|,\left|y_{1}-y_{2}\right|\right\} \quad .\left(M, d_{\Delta_{T}}\right) \quad$ is a metric space. For any point $p$, the ${ }^{\Delta} T_{\varepsilon}$ neighbourhood of $p$ is defined as $N_{d_{\Delta_{T}} \varepsilon}(p)=\left\{q ; d_{\Delta_{T}}(p, q)<\varepsilon+1\right\}$. Hence the ${ }^{\Delta} T_{1}$ neighbourhood of $p$ is defined as $N_{d_{\Delta_{T}}{ }^{1}}(p)=\left\{q ; d_{\Delta_{T}}(p, q)<2\right\}$ and the ${ }^{\Delta} T_{2}$ neighbourhood of $p$ is defined as $N_{d_{\Delta_{T^{\prime}}}}(p)=\left\{q ; d_{\Delta_{T}}(p, q)<3\right\}$. The ${ }^{\Delta} T_{1}$ neighbours of $p$ are $N_{d_{\Delta_{T^{1}}}}(p)-\{p\}$ and the ${ }^{\Delta} T_{2}$ neighbours of $p$ are $N_{d_{\Delta_{T^{2}}}}(p)-\{p\}$.

Definition 3.10

Grouping criterion:

Let $X$ be an image in levels of gray and $\Im$ a topology associated with $X$ and let $Y \subset \Im$. Let ' $d$ ' be a metric on $X$. Define $\varphi: Y x X \rightarrow R$ such that $\varphi((A, x))=m$ where $m$ is the maximum of the gray level difference between $x$ and the elements of $A$. Let $A \in Y$. Given a fixed $\varepsilon$ and $\delta$, an element $x \in X$ is said to belong to $A$ if $d(x, y)<\varepsilon+1$ for some $y \in A$ and $\varphi((A, x))<\delta$.

\section{Proposed Segmentation Algorithm}

\section{Left Triangular Algorithm}

Let $X$ be an image in levels of gray.

\section{Step I:}

From the histogram of the image, select the gray level of the region of interest to be segmented.

Step II:

Find the seed point of the region of interest. Here, the seed point is a point with maximum number of LT neighbours with gray level difference less than $\delta$ which is very small. If more than one point has the maximum number of LT neighbours then choose any one of those points as the seed point.

Let $A_{1}=\{x\}$ where $x$ is the seed point of the region of interest.

Step III:

Choose $\varepsilon=1$ and $\delta=2$. If $\exists$ a point $y \in X$ where $y \notin A_{1}$ such that $d_{L T}(x, y)<\varepsilon+1$ and $\varphi\left(\left(A_{1}, y\right)\right)<\delta$

then include $y$ in $A_{1}$ and rename it as $A_{2}$.

Repeat Step III again and again for the elements of $A_{2}$ till $\exists$ no point $y \in X$ satisfying the condition (1). That is, all the LT neighbours of $A_{1}$ are obtained.

\section{Step IV:}

If $\exists$ a point $y \in X$ where $y \notin A_{2}$ such that $d_{\Delta_{T}}(z, y)<\varepsilon+1$ where $z \in A_{2}$ and

$\varphi\left(\left(A_{2}, y\right)\right)<\delta$

then include $y$ in $A_{2}$ and rename it as $A_{3}$.

Repeat Step IV again and again for the elements of $A_{3}$ till $\exists$ no point $y \in X$ satisfying the condition (2). That is all the ${ }^{\Delta} T$ neighbours of $A_{2}$ are obtained.

Step V:

If $\exists$ a point $y \in X$ where $y \notin A_{3}$ such that $d_{\Delta^{T}}(z, y)<\varepsilon+1$ where $z \in A_{3}$ and $\varphi\left(\left(A_{3}, y\right)\right)<\delta$

then include $y$ in $A_{3}$ and rename it as $A_{4}$.

Repeat Step V again and again for the elements of $A_{4}$ till $\exists$ no point $y \in X$ satisfying the condition (3). That is all the ${ }_{\Delta} T$ neighbours of $A_{3}$ are obtained. 
The set $A_{4}$ is the segmented region of interest.

\section{Evaluation Measure}

Let $X$ be the set of pixels in the image. Define the ground truth $T \subset X$ as the set of pixels that were labeled as tumor by the expert. Similarly define the segmented tumor $S \subset X$ as the set of pixels that were labeled as tumor by the algorithm. $\bar{T}$ and $\bar{S}$ be the set of pixels that were labeled as non-tumor by the expert and algorithm respectively. The true positive $(T P)$ set is defined as $T P=T \cap S$, i.e., the set of pixels common to $T$ and $S$. i.e., the set of pixels that were labeled as tumor by the expert and algorithm. The true negative $(T N)$ set is defined as $T N=\bar{T} \cap \bar{S}$, i.e., the set of pixels common to $\bar{T}$ and $\bar{S}$. i.e., the set of pixels that were labeled as non-tumor by the expert and algorithm. The false negative $(F N)$ set is defined as $F N=T \cap \bar{S}$, i.e., the set of pixels common to $T$ and $\bar{S}$. i.e., the set of pixels that were labeled as tumor by the expert and nontumor by the algorithm. The false positive $(F P)$ set is defined as $F P=\bar{T} \cap S$, i.e., the set of pixels common to $\bar{T}$ and $S$. i.e., the set of pixels that were labeled as non-tumor by the expert and tumor by the algorithm.

The segmentation evaluation measure 'Accuracy' is defined as

$$
\text { Accuracy }=\frac{n(T P)+n(T N)}{n(T P)+n(T N)+\mathrm{n}(\mathrm{FP})+\mathrm{n}(\mathrm{FN})}
$$

\section{Experimental Work}

In this work, magnetic resonance images of brain affected by tumor are taken. The seed point of the region of interest is selected using the histogram of the image and metric topological $\varepsilon$ - neighbourhoods. The region of interest is grown from the seed point using the metric topological $\varepsilon$ neighbourhoods $N_{d_{L T}, \varepsilon}(p), N_{d_{\Delta_{T}} \varepsilon}(p), N_{d}{ }_{\Delta^{T}} \varepsilon(p)$ and the grouping criterion. The proposed region growing segmentation algorithm is implemented using MATLAB 7.0. The performance of the proposed algorithm is evaluated using the segmentation evaluation measure 'Accuracy'.

\section{Result Analysis And Discussion}

The magnetic resonance images of brain affected by tumor are segmented using the proposed Left Triangular Algorithm based on metric topological $\varepsilon$ - neighbourhoods and the grouping criterion.

Table 1 shows the accuracy values and the percentage of the quality of segmentation of the proposed Left Triangular Algorithm applied to magnetic resonance images of brain affected by tumor.

Fig 1 shows the ground truth images. Fig 2 shows the original magnetic resonance images of brain affected by tumor, the corresponding segmented region and the segmented region with boundary respectively using the proposed Left Triangular Algorithm.

\section{Conclusion}

In this work, a new region growing segmentation algorithm based on metric topological $\varepsilon$ neighbourhood and grouping criterion is introduced. To demonstrate the performance of the proposed Left Triangular Algorithm based on metric topological $\varepsilon$-neighbourhoods, the experiments have been conducted on magnetic resonance images of brain affected by tumor. The performance of the proposed segmentation algorithm is measured using the segmentation evaluation measure 'Accuracy'. The experimental results indicate that the percentage of the quality of segmentation by the proposed Left Triangular Algorithm is $98.59 \%$ or more. This work is a new metric topological approach for region growing segmentation of medical images to segment the region of interest.

\section{Table And Figures}

\begin{tabular}{|l|r|r|r|r|}
\hline EVALUATION MEASURE & IMAGE 1 & IMAGE 2 & IMAGE 3 & IMAGE 4 \\
\hline Accuracy Values & 0.9859 & 0.9892 & 0.9921 & 0.9952 \\
\hline Quality of segmentation in \% values & $98.59 \%$ & $98.92 \%$ & $99.21 \%$ & $99.52 \%$ \\
\hline
\end{tabular}

Table 1 - Accuracy and Quality 




Fig 1 - Ground Truth Images

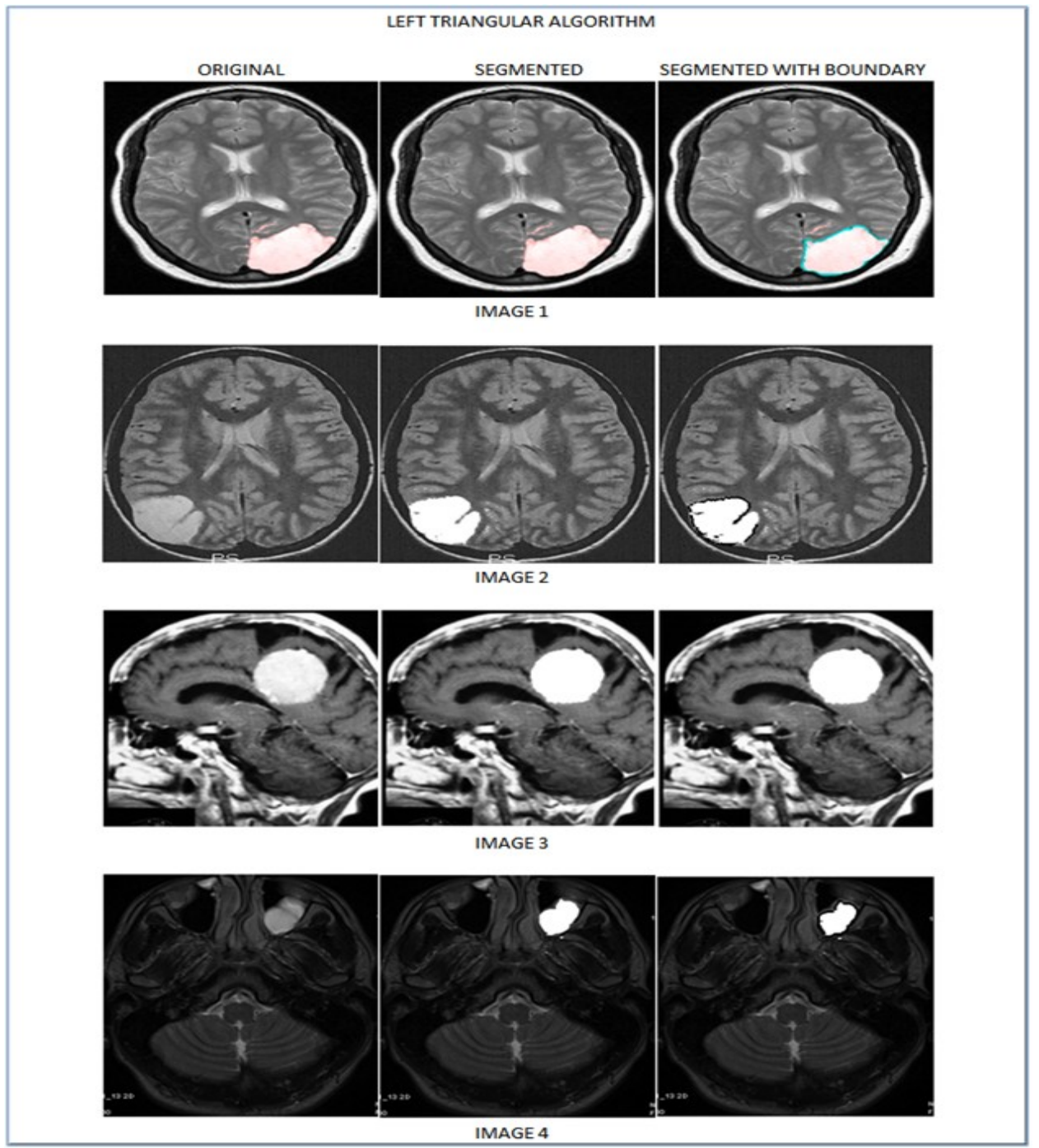

Fig 2 - Segmentation by Left Triangular Algorithm

\section{Acknowledgements}

The authors thank The University Grants Commission, India, to carry out this research work.

\section{References}

[1] Jiang Guangyou and Kang Gewen, A threshold segmentation algorithm based on neighbourhood characteristics, The tenth International conference on Electronic Measurement and Instruments ,IEEE , 2011,328-331.

[2] Chantal Revol and Michel Jourlin, A new minimum variance region growing algorithm for image segmentation, Pattern Recognition Letters, 18, 1997, 249-258.

[3] Yi-Ta Wu , Frank Y. Shih, Jiazheng Shi and Yih-Tyng Wu, A top-down region dividing approach for image segmentation, Pattern Recognition, 41, 2008, 1948-1960. 
[4] Maria Kallergi, Kevin Woods, Laurence P. Clarke, Wei Qian and Robert A. Clark, Image segmentation in digital mammography; Comparison of local thresholding and region growing algorithms, Computerized Medical Imaging and Graphics, Vol 16(5), 1992, 323-331.

[5] Pastore J., Bouchet A., Moler E. and Ballarin, V., Topological Concepts applied to Digital Image Processing, JCS\&T, Vol. 6, No. 2, 2006, 80-84.

[6] Gnanambal Ilango and R. Marudhachalam, Topological Concepts Applied to Image Segmentation, Journal of Advanced Studies in Topology, Vol. 4, No. 1, 2013,128-132.

[7] Tamas Sandor, David Metcalf and Young-Jo Kim, Segmentation of brain CT images using the concept of Region Growing, Int J Biomed Comput, 29, 1991, 133-147.

[8] Sang Uk Lee and Seok Yoon Chung, A comparative performance study of several global thresholding techniques for segmentation, Computer Vision, Graphics and Image processing, 52, 1990, 171 - 190.

[9] Junchai Gao , Zhiyong Lei , Zemin Wang and Keding Yan, Region growth segmentation based on dual - cores, Energy Procedia, 13, 2011, 4567-4571.

[10] M.M. Abdelsamea, An Automatic Seeded Region Growing for 2D Biomedical Image Segmentation, Proceedings of the International Conference on Environment and Bioscience, 21, 2011, 1-5.

[11] K. Martin Sagayam, V. Ayyappan, and S. Palani, Automatic Morphological Segmentation and Region Growing Method of Diagnosing Medical Images, International Journal of Information \& Computation Technology, Vol 2(6), 2012, $173-180$.

[12] Dinesh D. Patil and Sonal G. Deore , Medical Image Segmentation: A Review, International Journal of Computer Science and Mobile Computing, 2, 2013, 22-27.

[13] James R. Munkres, Topology (Prentice-Hall of India, 2007).

[14] R. Gonzalez, and R. Woods, Digital Image Processing (New York, Adison-Wesley, 1992).

[15] Gnanambal Ilango and B. Shanthi Gowri, $\varepsilon$ - Neighbourhood Median Filters to Remove Speckle Noise from CTImages, International Journal of Applied Information Systems, Vol. 4, No. 10, 2012, 40-46.

[16] R. Klette and A. Rosenfeld, Digital Geometry (San Fransisco, Kaufmann, 2004). 